\title{
Type 4 Phosphodiesterase Plays Different Integrating Roles in Different Cellular Domains in Pyramidal Cortical Neurons
}

\author{
Liliana R. V. Castro, ${ }^{1,2}$ Nicolas Gervasi, ${ }^{3}$ Elvire Guiot, ${ }^{1,2}$ Laetitia Cavellini, ${ }^{1,2}$ Viacheslav 0. Nikolaev, ${ }^{4}$ \\ Danièle Paupardin-Tritsch, ${ }^{1,2}$ and Pierre Vincent ${ }^{1,2}$ \\ ${ }^{1}$ Centre National de la Recherche Scientifique, Unité Mixte de Recherche (UMR) 7102, Paris, France, 2Université Pierre et Marie Curie, UMR 7102, Paris, \\ France, ${ }^{3}$ ESPCI (École Supérieure de Physique et de Chimie Industrielles de la Ville de Paris), UMR 7637, Paris, France, and ${ }^{4}$ Institute of Pharmacology \\ and Toxicology, University of Würzburg, D-97078 Würzburg, Germany
}

We investigated the role of phosphodiesterases (PDEs) in the integration of cAMP signals and protein kinase A (PKA) activity following $\beta$-adrenergic stimulation, by carrying out real-time imaging of male mouse pyramidal cortical neurons expressing biosensors to monitor cAMP levels (Epac1-camps and Epac2-camps300) or PKA activity (AKAR2). In the soma, isoproterenol (ISO) increased the PKA signal to approximately half the maximal response obtained with forskolin, with a characteristic $\beta_{1}$ pharmacology and an $\mathrm{EC}_{50}$ of $4.5 \mathrm{~nm}$. This response was related to free cAMP levels in the submicromolar range. The specific type 4 PDE (PDE4) inhibitor rolipram had a very small effect alone, but strongly potentiated the PKA response to ISO. Blockers of other PDEs had no effect. PDE4 thus acts as a brake in the propagation of the $\beta_{1}$-adrenergic signal from the membrane to the bulk somatic cytosol. The results for a submembrane domain were markedly different, whether recorded with a PKA-sensitive potassium current related to the slow AHP or by two-photon imaging of small distal dendrites. The responses to ISO were stronger than in the bulk cytosol. This is consistent with the cAMP/PKA signal being strong at the membrane, as shown by electrophysiology, and favored in cellular domains with a high surface area to volume ratio, in which this signal was detected by imaging. Rolipram alone also produced a strong cAMP/PKA signal, revealing tonic cAMP production. PDE4 thus appears as a crucial integrator with different physiological implications in different subcellular domains.

\section{Introduction}

Neuromodulators control various properties of neurons. Norepinephrine, one of the many neuromodulatory substances affecting diverse aspects of behavior, is known to play an important role in the cortex (Sara, 2009). Norepinephrine exerts its action partly through $\beta$-adrenergic receptors $(\beta$-AR), through which it stimulates the production of intracellular cAMP. The cAMP and cAMP-dependent protein kinase (PKA) signaling cascade is thus an important integrator of neuromodulatory signals, and dysfunctions in this signaling pathway are associated with various psychiatric conditions.

Phosphodiesterases are widely thought to play a crucial role, by degrading cAMP into AMP, thereby terminating the response. However, the contribution of phosphodiesterases to the precise dynamics of signal integration remains poorly understood. Various phosphodiesterases are expressed in the brain, but type 4 phosphodiesterases (PDE4) have a critical function. Mice lacking the PDE4D gene behave as if they had been treated with antidepressants (Zhang et al., 2002). Chromosomal translocations in the PDE4B gene (Millar et al., 2005) or alterations in the DISC1

\footnotetext{
Received Nov. 18, 2009; revised March 23, 2010; accepted March 26, 2010.

This work was supported by Centre National de la Recherche Scientifique, Université Pierre et Marie Curie, Fédération pour la Recherche sur le Cerveau, and Agence Nationale de la Recherche.

Correspondence should be addressed to Pierre Vincent, Intégration Cellulaire des Signaux Neuromodulateurs, UMR7102 Neurobiologie des Processus Adaptatifs, CNRS UPMC, 9, quai St Bernard, F-75005 Paris, France. E-mail: pierre.vincent@upmc.fr.

DOI:10.1523/JNEUROSCI.5851-09.2010

Copyright $\odot 2010$ the authors $\quad 0270-6474 / 10 / 306143-09 \$ 15.00 / 0$
}

gene (Millar et al., 2000), which encodes a protein known to bind PDE4B, are also associated with psychiatric illnesses (Millar et al., 2007). In addition, the cAMP produced in response to isoproterenol (ISO) is mostly degraded by PDE4 in the cortex (Ye and O'Donnell, 1996). These studies have shown that PDE4 activity is required for the correct integration of neuromodulatory signals. This is further confirmed by the effects of rolipram, a specific PDE4 blocker, which reverts deficits observed in several psychiatric disorders (Wachtel, 1983; Zhang et al., 2002) related with changes in cAMP (O'Donnell and Zhang, 2004; H. T. Zhang et al., 2005).

The side effects of rolipram preclude its use in medicine, but it would nonetheless be interesting to understand the cellular basis of its action. This question can now be addressed directly by cellular imaging methods. Genetic constructs encoding fluorescent resonance energy transfer (FRET) probes for the cAMP/PKA signaling cascade (Vincent et al., 2008) have been used for the real-time measurement of PKA activity with AKAR2 (J. Zhang et al., 2005) and cAMP levels have been assessed with Epac-derived sensors (Nikolaev et al., 2004; Norris et al., 2009), from the cell body to small dendritic branches in pyramidal cortical neurons in brain slices. PKA activity has also been monitored with the slow afterhyperpolarization potential (sAHP), which is suppressed by PKA in response to several neuromodulators (Haas and Konnerth, 1983; Lancaster and Adams, 1986; Nicoll, 1988; Sah, 1996). We found that cAMP/PKA signaling was tightly controlled by PDE4 in both cellular domains, but with very different consequences for signal integration. 
In the soma, PDE4 strongly limited the cAMP/PKA signals elicited by $\beta 1-\mathrm{AR}$. By contrast, in a domain in which recordings were made by two-photon imaging in small dendrites or by electrophysiology, PDE4 continuously degraded cAMP which was tonically produced. PKA activity was thus maintained at a level such that both up- and downregulations were possible, as illustrated by potassium current measurements.

\section{Materials and Methods}

Virus production, brain slices, and infection. We used the Sindbis virus as a vector (Ehrengruber et al., 1999). The coding sequences of AKAR2, AKAR2mut, Epac1-camps and Epac2-camps300 were subcloned into the viral vector pSinRep5 (Invitrogen), as previously described (Gervasi et al., 2007). The Q69M substitution was introduced into the EYFP acceptor fluorophore of Epac2-camps300, to convert the acceptor into a Citrine. The resulting construct, Epac2-camps300-Cit, was used for twophoton imaging in dendrites. Male 9- to 14 -d-old C57BL/6 mice (Janvier) were used in this study. Coronal brain slices were cut with a HM650V vibratome (Microm), or with a VT1000S or VT1200S microtome (Leica). Slices were prepared in an ice-cold solution of the following composition (in $\mathrm{mm}$ ): $125 \mathrm{NaCl}, 0.4 \mathrm{CaCl}_{2}, 1 \mathrm{MgCl}_{2}, 1.25$ $\mathrm{NaH}_{2} \mathrm{PO}_{4}, 26 \mathrm{NaHCO}_{3}$ and 25 glucose, saturated with $5 \% \mathrm{CO}_{2}$ and $95 \%$ $\mathrm{O}_{2}$. The slices were incubated in this solution for $30 \mathrm{~min}$ and then placed on a Millicell-CM membrane (Millipore) with culture medium (50\% minimal essential medium, 50\% Hanks' balanced salt solution, $6.5 \mathrm{~g} / \mathrm{L}$ glucose, penicillin-streptomycin, Invitrogen). The viral vector $(\sim 5 \times$ $10^{5}$ particles per slice) was added and slices were incubated overnight at $35^{\circ} \mathrm{C}$ with $5 \% \mathrm{CO}_{2}$. Before recording, slices were incubated for $1 \mathrm{~h}$ in the recording solution (identical to the solution used for cutting except that the calcium concentration was $2 \mathrm{~mm}$ ). During recordings, brain slices were continuously perfused at $32^{\circ} \mathrm{C}$ with this solution saturated with $5 \% \mathrm{CO}_{2} /$ $95 \% \mathrm{O}_{2}$, as previously described (Gervasi et al., 2007). Each slice was used to test one agonist only and slices were discarded after the forskolin (FSK), or FSK+IBMX (3-isobutyl-1-methylxanthine) response.

Optical recordings on brain slices. Recordings were made on visually identified pyramidal neurons in layer $\mathrm{V}$ of the mouse parietal cortex. Wide-field images were obtained with an Olympus BX50WI or BX51WI upright microscope with a $20 \times 0.5$ numerical aperture $(\mathrm{NA})$ or a $40 \times 0.8$ NA water-immersion objective and either a Micromax digital camera (Roper Scientific) or an ORCA-AG camera (Hamamatsu). Acquisition was controlled using iVision (BioVision). The excitation and dichroic filters used were D436/20 and 505dclp. Signals were acquired by alternating the emission filters, HQ480/40 for CFP and D535/40 for YFP, with a filter wheel (Sutter Instruments). All filters were obtained from Chroma Technology.

Two-photon images were obtained with a custom-built two-photon laser scanning microscope based on an Olympus BX51WI upright microscope with a $60 \times 0.9$ NA water-immersion objective and a Ti:sapphire laser (MaiTai HP; Spectra Physics) tuned to $850 \mathrm{~nm}$ for CFP excitation. Galvanometric scanners (model 6210, Cambridge Technology) were used for raster scanning, and a piezo-driven objective scanner (P-721 PIFOC; Physik Instrumente GmbH) was used for Z-Stack image acquisition. The system was controlled by MPscope software (Nguyen et al., 2006). A two-photon emission filter was used to reject residual excitation light (E700 SP, Chroma Technology). A fluorescence cube containing 479/40 and 542/50 emission filters and a $506 \mathrm{~nm}$ dichroic beamsplitter (FF01-479/40, FF01-542/50, and FF506-Di02-25x36 Brightline Filters; Semrock) was used for the orthogonal separation of the two fluorescence signals. Two imaging channels (H9305 photomultipliers, Hamamatsu) were used for simultaneous detection of the two types of fluorescence emission.

Images were analyzed with custom routines written in the IGOR Pro environment (Wavemetrics). For AKAR2, we calculated the ratio of each pixel as the emission at $535 \mathrm{~nm}$ divided by the emission at $480 \mathrm{~nm}$ (F535/F480). This ratio was inverted for Epac2-camps300 and Epac1camps. The pseudocolor images were calculated to display simultaneously the ratio information (coded in hue) and the fluorescence of the preparation (averaged F480 and F535; coded in intensity).
Patch-clamp recordings. Patch pipettes (2-3 M $\Omega$ ) were filled with a solution containing $130 \mathrm{~mm}$ potassium methane sulfonate, $10 \mathrm{~mm}$ HEPES, 1 mm 5,5'-dibromo BAPTA, 5 mm $\mathrm{MgCl}_{2}, 0.1 \mathrm{~mm} \mathrm{CaCl}_{2}, 4 \mathrm{~mm}$ ATP- $\mathrm{Na}_{2}, 5 \mathrm{~mm}$ creatine phosphate, $\mathrm{pH}$ 7.35. Potentials were corrected for the liquid junction potential $(7 \mathrm{mV})$. Cells were maintained at holding potential of $-67 \mathrm{mV}$. The amplitude of the sAHP current was monitored by applying a train of 20 steps of $3 \mathrm{~ms}$ duration at a frequency of 80 $\mathrm{Hz}$, from $-67 \mathrm{mV}$ to $+13 \mathrm{mV}$, but the amplitude of the sAHP current was less stable than the holding current, which was therefore used in our measurements and called tonic potassium current (tonic $I_{\mathrm{K}}$ ). A $10 \mathrm{mV}$ hyperpolarizing jump was applied before each train, to monitor series and input resistances. Experiments were discarded if the series resistance increased above $10 \mathrm{M} \Omega$. The transient capacitive currents remained constant throughout the experiments. Current-clamp recordings were performed with the same internal solution, except that 5,5'-dibromo BAPTA was replaced by EGTA (1 mM). Neurons were held at $-65 \mathrm{mV}$ and current pulses were applied until trains of spikes were obtained. All data were acquired with an Axopatch 200B amplifier (Molecular Devices) and analyzed with AxographX software (AxoGraph Scientific).

Data analysis and statistics. In each slice, for each neuron, the response was normalized with respect to the maximal ratio change obtained with FSK (AKAR2) or FSK+IBMX (Epac1-camps and Epac2-camps300). We analyzed the responses of at least 4 the neurons in each slice, to generate one data point. The Hill equation was fitted to the concentrationresponse curve for the effects of ISO. The amplitude of tonic potassium current was measured as the difference between basal and FSK inhibited tonic current. All data are expressed as means \pm SEM and the number of slices is denoted by $n$. When appropriate, unpaired Student's $t$ tests were used for statistical comparisons and a $p$ value $<0.05$ was considered statistically significant and indicated by an asterisk.

Drugs. ISO, IBMX, and BRL 50481 were purchased from SigmaAldrich FSK, ICI 118551 [( \pm )-1-[2,3-(dihydro-7-methyl- $1 H$-inden-4-yl) oxy]-3-[(1-methylethyl)amino]-2-butanol], CGP 20712 [[2-(3-carbamoyl4-hydroxyphenoxy)-ethylamino]-3-[4-(1-methyl-4-trifluormethyl2-imidazolyl)-phenoxy]-2-propanolmethanesulfonate] dihydrochloride, and apamine were obtained from Tocris Bioscience; Rp-cAMPS was obtained from Biolog, and 5,5'-dibromo BAPTA was obtained from Invitrogen.

\section{Results}

\section{$\boldsymbol{\beta}$-AR stimulation increases PKA activity via $\boldsymbol{\beta}_{1}$-AR}

In the first series of experiments, we studied the CAMP/PKA response to the stimulation of $\beta$-AR. We investigated whether AKAR 2 could be used as a reporter of the activation of PKA by the nonselective $\beta$-AR agonist ISO. Bath application of ISO (100 nM) induced a clear increase in the F535/F480 emission ratio in all AKAR2-expressing cells (Fig. 1). ISO increased the FRET emission ratio to $43 \pm 4 \%(n=11)$ of the maximal response induced by the direct adenylyl cyclase activator FSK (always used at 13 $\mu \mathrm{M})$. The response decreased with prolonged exposure to the agonist, probably as a consequence of receptor desensitization. FSK led to maximal phosphorylation of the sensor, because the broad-spectrum PDEs inhibitor IBMX produced no further increase in the ratio above that observed with FSK (Gervasi et al., 2007). All measurements were therefore normalized with respect to the FSK response obtained at the end of each experiment.

We checked that the signal recorded in our conditions was dependent on the phosphorylation of AKAR2, by carrying out the same experiments with a mutant form of AKAR2 (AKAR2mut), in which the threonine residue that is phosphorylated to activate the molecule was replaced by an alanine residue (J. Zhang et al., 2005; Gervasi et al., 2007). In these conditions, no change in emission ratio was observed with $1 \mu \mathrm{M}$ ISO or FSK $(n=4)$.

Both $\beta_{1}$ - and $\beta_{2}$-AR are positively coupled with adenylyl cyclase via $\mathrm{Gs}$ proteins. The non selective activation of $\beta$-AR had a dose-dependent effect on AKAR2 emission ratio, with an $\mathrm{EC}_{50}$ of 
A
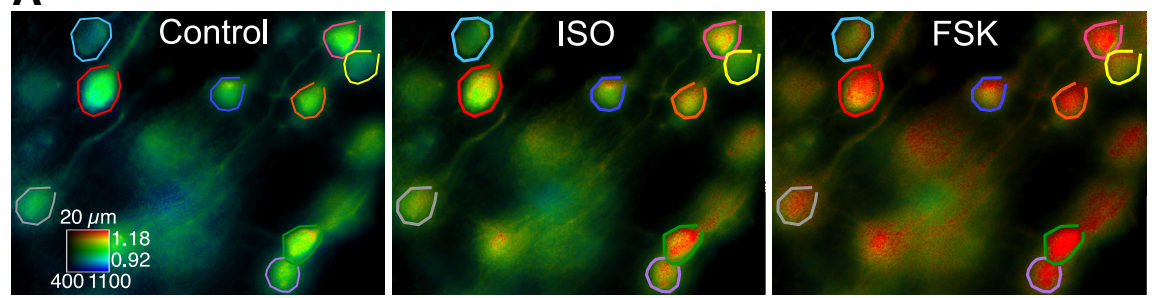

B

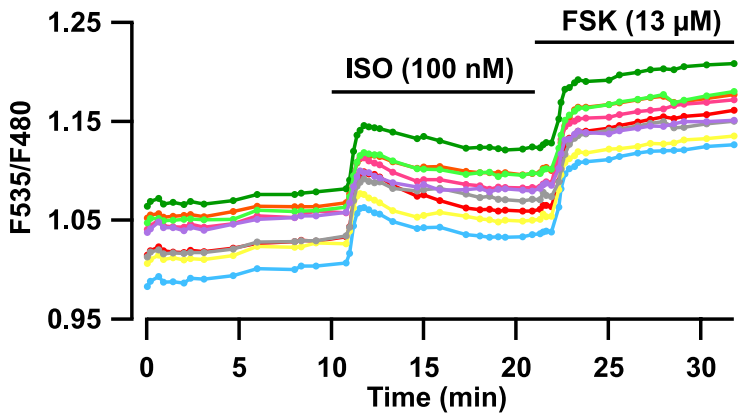

Figure 1. $\quad \beta$-adrenergic receptor stimulation activates PKA in the parietal cortex. $\boldsymbol{A}$, Pseudocolor images representing the AKAR2 fluorescence emission ratio indicative of PKA-dependent phosphorylation, in control conditions (left), in the presence of $100 \mathrm{~nm}$ isoproterenol (ISO, center) and $13 \mu \mathrm{m}$ forskolin (FSK, right). The calibration square indicates fluorescence intensity (in counts/pixel/s) horizontally and F535/F480 ratio vertically. $\boldsymbol{B}$, Each trace indicates the F535/F480 emission ratio measured on the regions indicated by the color contour drawn on the images. ISO (100 nм) and FSK (13 $\mu \mathrm{m})$ were added to the bath for the time period indicated by the bar.

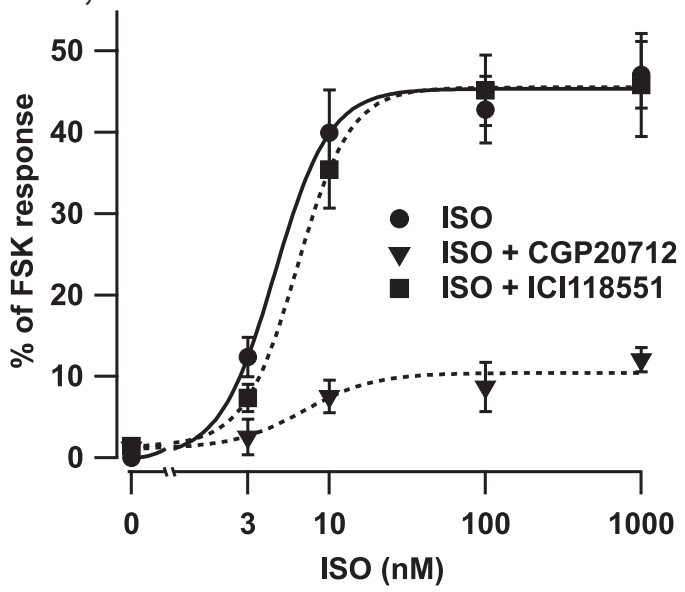

Figure 2. $\quad \beta_{1}$-AR stimulation increases AKAR2 fluorescence emission ratio in cortical neurons in a dose-dependent manner. Cortical brain slices were challenged with the nonselective $\beta$-AR agonist, ISO, alone or in the presence of $\beta_{1}$ - or $\beta_{2}$-AR antagonists (CGP 20712 (100 nM) and ICI $118551(10 \mathrm{~nm})$, respectively. For each neuron, the response to ISO is normalized with respect to the response to forskolin. Each data point corresponds to at least 7 independent experiments.

$4.5 \mathrm{~nm}$ (Fig. 2). We used a combination of ISO and the $\beta_{2}$-AR antagonist ICI $118551(10 \mathrm{nM})$ to stimulate $\beta_{1}$-AR selectively, and a combination of ISO and the $\beta_{1}$-AR antagonist CGP 20712 (100 $\mathrm{nM}$ ) to stimulate $\beta_{2}$-AR. The specific activation of $\beta_{1}$-AR (ISO with ICI 118551) resulted in a dose-dependent effect similar to that observed with ISO alone $\left(\mathrm{EC}_{50}\right.$ of $\left.5.9 \mathrm{nM}\right)$. By contrast, in the presence of CGP 20712, ISO had only a small effect (22.8\% of the response to ISO alone), indicating that ISO activates PKA in cortical neurons principally via the $\beta_{1}$-AR subtype.

\section{$\beta$-AR stimulation results in low levels of cAMP production} The cAMP signal underlying the PKA response was then measured with a CAMP sensor consisting of the cAMP-binding domains of Epac1, Epac1-camps, which has an $\mathrm{EC}_{50}$ of $2.4 \mu \mathrm{M}$
(Nikolaev et al., 2004). Surprisingly, we observed no significant response to ISO (Fig. 3A-C), whereas FSK alone increased the emission ratio to just over half $(60 \pm$ $6 \% ; n=4)$ the maximal response obtained with FSK plus $200 \mu \mathrm{M}$ IBMX. Thus, even FSK is not sufficient for the maximal activation of Epac1-camps. We then used a new, more sensitive cAMP sensor, Epac2-camps300 which has an $\mathrm{EC}_{50}$ of $320 \mathrm{nM}$ for cAMP, in the range of concentrations relevant for PKA activation (Norris et al., 2009). Bath application of ISO (100 nM) induced a significant increase in the F480/F535 emission ratio in all Epac2camps300-expressing neurons (Fig. 3D-F). FSK produced a near-maximal response, as the further addition of IBMX $(200 \mu \mathrm{M})$ increased the F480/F535 emission ratio by $<20 \%$, consistent with a higher sensitivity to cAMP than Epacl-camps. We therefore used this sensor for quantification purposes, calculating the response obtained as a percentage of the maximal FSK+IBMX response. With Epac2-camps300, 100 nM ISO induced a response that was $22 \pm 3 \%(n=$ 10) of FSK + IBMX, suggesting that the increase in free CAMP concentration in response to ISO remains below the $\mathrm{EC}_{50}$ of $320 \mathrm{~nm}$.

\section{PDE4 inhibition potentiates the cytosolic cAMP/PKA response induced by $\boldsymbol{\beta}$-AR}

The amplitude of the AKAR2 response to a saturating ISO concentration was only half the maximal response to FSK $(46 \pm 6 \%$; $n=8$; Fig. 2). Among the various mechanisms that may limit this PKA activation we suspected the involvement of PDE, including PDE4. Indeed, previous studies have shown that the inhibition of PDE4 activity increases the global levels of cAMP produced in response to the stimulation of $\beta$-AR in homogenates of slices of mouse cerebral cortex (Yamashita et al., 1997; H. T. Zhang et al., 2005). We investigated the effect of rolipram, a specific blocker of the PDE4 family, on AKAR2 phosphorylation level. Rolipram $(100 \mathrm{nM})$ treatment alone resulted in a significant, but small increase in PKA activity ( $5.9 \pm 1.2 \%$ of the maximal FSK response; $n=21$; Fig. $4 A$ ). However, rolipram significantly enhanced the PKA responses to ISO: rolipram increased ISO responses by $300 \%$ for low concentrations ( $3 \mathrm{~nm}$ ) and $40 \%$ for saturating concentrations $(1 \mu \mathrm{M})$.

In the presence of the $\beta_{1}$-AR inhibitor CGP (100 nM), rolipram plus $1 \mu \mathrm{M}$ ISO resulted in a response with an amplitude $24 \pm$ $4 \%(n=4)$ that of the maximal response obtained with FSK, whereas, in the absence of rolipram, the same concentration of ISO induced a response $13 \pm 2 \%(n=10)$ the size of the maximal response obtained with FSK. These findings indicate that the potentiating effect of rolipram is exerted principally through the $\beta_{1}$-AR receptor (data not shown).

We thought that this effect might result from regulation at the level of cAMP concentration. Modeling studies and recordings on primary cultures of embryonic hippocampal neurons indicated that ISO stimulation in the presence of rolipram results in a strong increase in cAMP levels (Neves et al., 2008). This was tested in our more mature preparation of cortical brain slices, with the cAMP sensor Epac2-camps300. Rolipram had negligible 
effects on its own, but doubled the effects of $100 \mathrm{~nm}$ ISO (Fig. $4 \mathrm{~B}$ ), increasing the amplitude of the response from $22 \pm 3 \%$ $(n=10)$ (see above) to $41 \pm 2 \%(n=8)$. Thus, PDE4 does indeed regulate the $\beta_{1}$-AR response in cortical pyramidal neurons, at the level of cAMP concentration, which remains below micromolar levels even when PDE4 is inhibited.

However, PDE4 may not be the only PDEs regulating the cAMP signal. We tested this hypothesis by investigating the role of other PDEs, using a combination of several PDEs inhibitors ( $1 \mu \mathrm{M}$ cilostamide, $10 \mu \mathrm{M}$ EHNA, $10 \mu \mathrm{M}$ zaprinast and $1 \mu \mathrm{M}$ BRL50481) to inhibit PDE1, PDE2, PDE3, PDE5 and PDE7. This combination had no significant effect on the AKAR2 response to $100 \mathrm{~nm}$ ISO (44 $\pm 6 \%$; $n=6$ ). Based on these findings we conclude that, in pyramidal neurons, PDE4 are the major PDEs hydrolyzing the cAMP produced in response to $\beta_{1}$-AR activation. PDEs other than PDE4 and/or cAMP extrusion through multidrug resistance protein, for example, must nevertheless be active, because, even in the presence of rolipram, the response to ISO never reached the maximal level obtained with forskolin.

\section{Tonic adenylyl cyclase and PDE4}

activities in small dendrites

Wide-field imaging reports changes in cAMP concentration or PKA activity level mainly in the somatic cytosol. However, the cAMP/PKA cascade has been reported to differ at the membrane compartment in cultured cells (Rich et al., 2001) and in intralaminar thalamic neurons (Gervasi et al., 2007). Modeling studies have also shown that, with their higher surface area to volume ratio, dendrites with a small diameter should have different cAMP dynamics (Neves et al., 2008). Using two-photon microscopy on cortical brain slice preparations, we recorded AKAR2 ratio changes in layer $\mathrm{V}$ pyramidal neurons in the cell body and in small dendritic branches emanating from these neurons and recorded in layer I.

In the cell body, ISO (100 nM) increased the ratio to $60 \%$ ( $n=$ 3) of the FSK response, a response similar to that obtained with wide-field imaging. However, responses of small dendrites were significantly larger with $93 \pm 5 \%(n=3)$ of the FSK control (Fig. $5 C)$. Thus, small dendrites do indeed behave differently from the cell body. We then assessed the effect of PDE4 inhibition: rolipram alone increased the AKAR2 ratio to $35 \pm 10 \%(n=4)$ of the FSK response (Fig. $5 A, B$ ), a much larger response than that recorded by wide-field imaging over cell bodies.

We checked that this increase in AKAR response resulted from an increase in cAMP concentration, by recording the cAMP signal in small dendrites with Epac2-camps300-Cit, a derivative of Epac2-camps300 in which EYFP is replaced by the more stable citrine. Rolipram alone increased the Epac2-camps300-Cit ra-
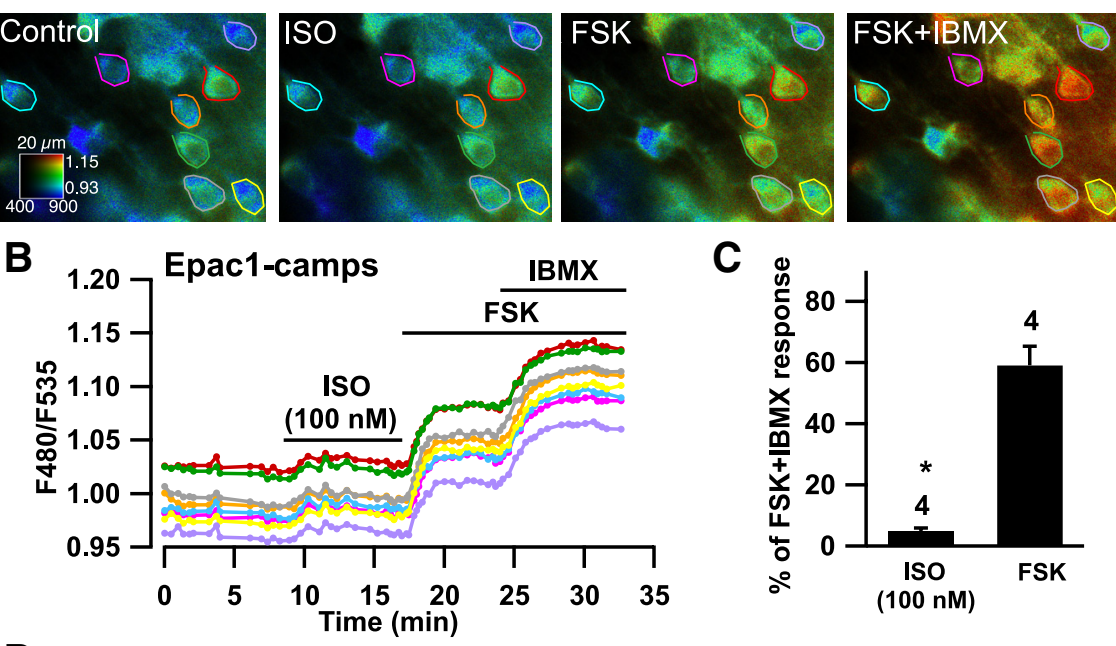

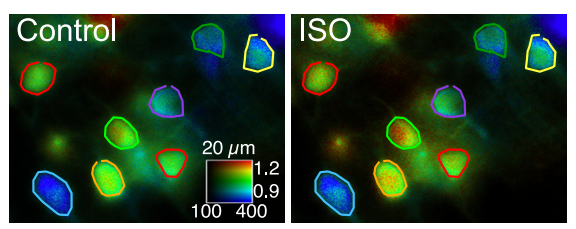

E

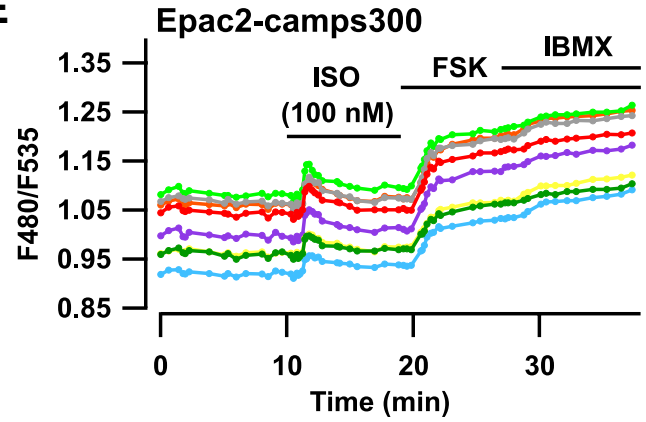

$\mathbf{F}$
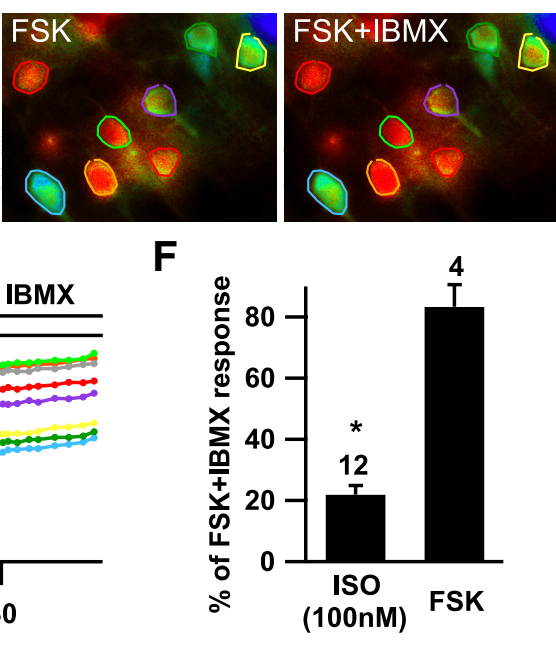

Figure 3. Epac1-camps reported no significant cAMP signal in response to $\beta$-AR stimulation, whereas the more sensitive Epac2-camps300 did. $\boldsymbol{A}, \boldsymbol{D}$, Pseudocolor images presenting fluorescence emission ratios indicative of changes in cAMP concentration, as measured with Epac1-camps $(\boldsymbol{A})$ and Epac2-camps300 (D), in the presence of ISO, FSK, and FSK + IBMX. $\boldsymbol{B}$, E, Each trace

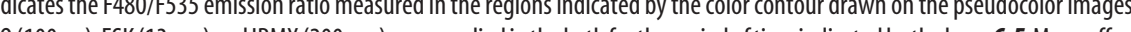
O (100 nM), FSK (13 $\mu \mathrm{M})$ and IBMX (200 $\mu \mathrm{M})$ were applied in the bath for the period of time indicated by the bars. $C, F$, Mean effect of ISO and FSK on the ratio change in Epac1-camps- and Epac2-camps300-expressing neurons, expressed as the percentage of the maximal response obtained with FSK + IBMX. The asterisk indicates a statistically significant difference.

tio to $44 \pm 4 \%(n=4)$ of the maximal FSK + IBMX response (Fig. $5 D, E)$. The application of ISO $(100 \mathrm{~nm})$, alone or in the presence of rolipram, strongly increased the ratio to $67 \pm 4 \%$ $(n=4)$ and $92 \pm 3 \%(n=4)$ of the maximal FSK+IBMX response, respectively (Fig. $5 F$ ). Again, this response was much larger than the response recorded by wide-field imaging over cell bodies (Fig. 4B).

Thus, ISO stimulation produces a stronger response in small dendrites than in the cell body. In addition, adenylyl cyclases tonically produce cAMP, which is degraded by PDE4, resulting in the maintenance of low resting concentrations.

PDE4 controls the phosphorylation level of the potassium current associated with sAHP

We then investigated the impact of this cAMP/PKA signaling on neuronal activity. The slow afterhyperpolarization (sAHP) current regulates neuronal excitability in the cortical pyramidal neurons of rodents (McCormick and Prince, 1986) and humans 


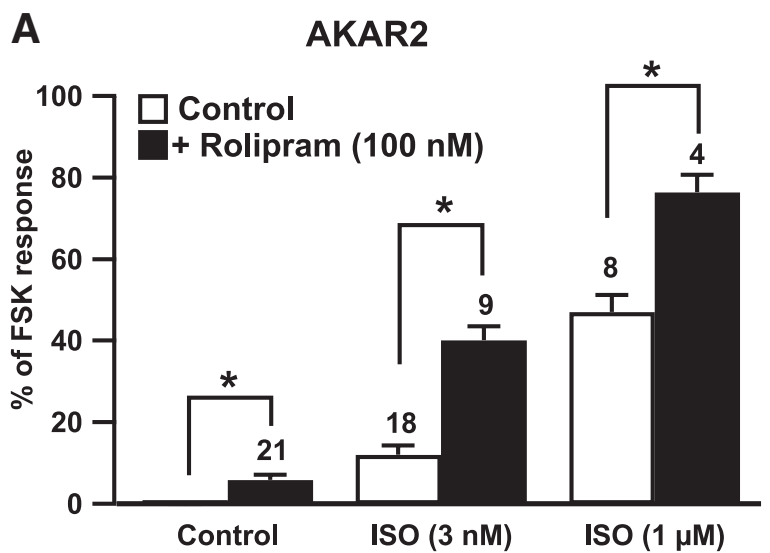

B EPAC2-camps300

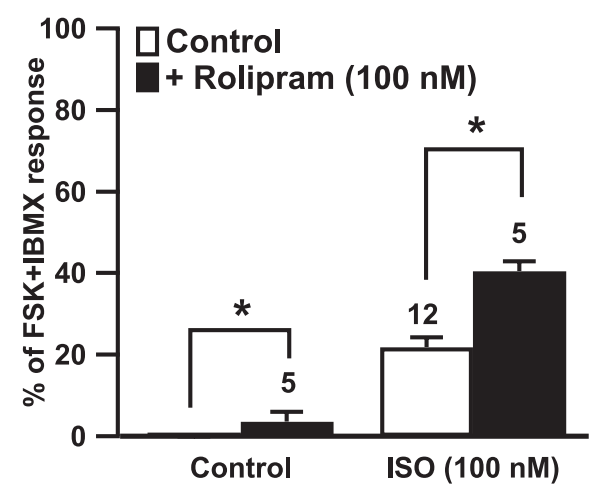

Figure 4. PDE4 inhibition with rolipram potentiates the $\beta$-AR response in cortical neurons expressing AKAR2 and Epac2-camps300. $A, B$, Mean effect of the $\beta$-AR agonist ISO, measured with AKAR2 $(\boldsymbol{A})$ or the Epac2-camps300 sensor $(\boldsymbol{B})$, tested alone or in the presence of rolipram. The mean ratio change in both sensors is expressed as a percentage of the maximal response obtained with FSK (AKAR2) or FSK + IBMX (Epac2-camps300). The asterisk indicates a statistically significant difference.

(McCormick and Williamson, 1989). This current, the molecular identity of which remains unknown, is associated with a tonic outward potassium current (referred to hereafter as tonic $I_{\mathrm{K}}$ ), and both currents are strongly reduced by PKA-mediated phosphorylation in response to various neuromodulators (Goaillard and Vincent, 2002). We therefore used the tonic $I_{\mathrm{K}}$ to investigate PKA activation at the plasma membrane and as an indicator of the effect of PKA on neuronal excitability.

We recorded the sAHP current in pyramidal cortical neurons, with an associated tonic $I_{\mathrm{K}}$ of $<100 \mathrm{pA}$; both the sAHP current and tonic $I_{\mathrm{K}}$ were suppressed by FSK. Replacement of the EGTA in the internal solution by $1 \mathrm{mM} 5,5$-dibromo BAPTA has been reported to potentiate tonic $I_{\mathrm{K}}$ strongly, while maintaining its sensitivity to PKA (Lancaster and Batchelor, 2000; Lancaster et al., 2006). Under these conditions, tonic $I_{\mathrm{K}}$ had an amplitude of $460 \pm 40 \mathrm{pA}(n=22)$ and was observed in $<80 \%(n=54)$ of the tested pyramidal neurons. Apamin $(100 \mathrm{~nm} ; n=4)$ had no effect on this current, demonstrating a lack of involvement of SK channels. This current was used to quantify PKA activity at the plasma membrane in response to various drugs.

We first examined the effect of PDE4 on tonic $I_{\mathrm{K}}$. Rolipram (100 nM) alone strongly inhibited this current (Fig. 6A) by a mean of $225 \pm 42 \mathrm{pA}$ ( $55 \%$ decrease; $n=8$ ). This is consistent with the constitutive synthesis of cAMP, which is tightly controlled by PDE4, as revealed by imaging experiments in small dendrites.
The application of ISO $(10 \mathrm{~nm})$ in the presence of rolipram strongly reduced the remaining current to $37 \pm 22 \mathrm{pA}(n=5)$, and subsequent application of FSK $(13 \mu \mathrm{M})$ only left a small leak current of $-55 \pm 27 \mathrm{pA}(n=19$; Fig. $6 A)$. In the absence of rolipram, low concentrations of ISO ( $1 \mathrm{nM})$ clearly inhibited tonic $I_{\mathrm{K}}(58 \%)$, with higher concentrations (10 nM) having an even stronger inhibitory effect $(84 \%)$. In the presence of rolipram, the inhibitory effect of ISO was potentiated to $75 \%(n=7)$ and $92 \%(n=5)$, respectively (Fig. $6 B)$.

For confirmation of the involvement of PKA in the inhibitory effect of rolipram, we performed the same experiments with an intracellular solution containing $500 \mu \mathrm{M}$ Rp-cAMPS, a specific PKA inhibitor. Rp-cAMPS alone doubled the tonic $I_{\mathrm{K}}$ to $875 \pm 71$ pA $(n=4)$, confirming the tight control of this current by constitutive PKA activity (Fig. $6 \mathrm{~B}$ ). In these conditions, neither rolipram alone (100 nM) nor rolipram with $10 \mathrm{~nm} \mathrm{ISO} \mathrm{inhibited} \mathrm{tonic}$ $I_{\mathrm{K}}$, demonstrating the exclusive role of PKA in modulating tonic $I_{\mathrm{K}}$. This experiment also demonstrated that rolipram did not function as a direct blocker of the channel underlying tonic $I_{\mathrm{K}}$.

The effect of rolipram on tonic $I_{\mathrm{K}}$ was also observed when 1 mM BAPTA was used as the internal calcium buffer $(n=7)$, ruling out a possible nonspecific effect of 5,5'-dibromo BAPTA on the cAMP/PKA signaling cascade.

\section{PDE4 inhibition promotes excitability in pyramidal cells}

The sAHP current and associated tonic $I_{\mathrm{K}}$ are small with standard intracellular solution, but they nonetheless have profound impact on the resting membrane potential and spike pattern, suggesting that PDE4 may play a crucial role in the control of electrical activity in pyramidal neurons. This was shown by patch-clamp recordings in current-clamp mode: rolipram increased the number of spikes obtained during the depolarizing current pulse ( $n=5$, Fig. 7 ).

\section{Discussion}

The recent advent of biosensors have opened up a new field of research in cellular neurosciences, allowing real-time quantitative imaging at the subcellular level in individual neurons in brain slice preparations. The use of biosensors in combination with electrophysiology made it possible to reconsider the importance of PDE4 in the regulation of signal integration in morphologically intact cortical pyramidal neurons. Our results reveal two different functional compartments: the bulk cytosol, which can be monitored by imaging cell bodies, and a submembrane domain, which can be studied by recording the PKA-sensitive tonic $I_{\mathrm{K}}$ or by imaging small dendrites. The bulk cytosol and submembrane domain differ in the amplitude of their response to receptor stimulation. PDE4 plays a crucial role in both domains, but the physiological consequences of its action differ between the two domains.

\section{Compartmentation of the signals: bulk cytosol versus} submembrane domain

In the bulk cytosol, the stimulation of $\beta_{1}$-AR receptors induced responses barely half as strong as the maximal response, demonstrating an attenuation of the signal during its journey from the membrane. PDE4 is involved in this attenuation, because its inhibition strongly potentiated the CAMP/PKA signal obtained in response to the activation of $\beta_{1}$-AR receptors. In addition, PDE4 had little effect on resting PKA activity level, showing that the bulk cytosol is little affected by the tonic cAMP production.

The cAMP signal was processed differently in the submembrane domain as reported by electrophysiology. Tonic $I_{\mathrm{K}}$ was 
A
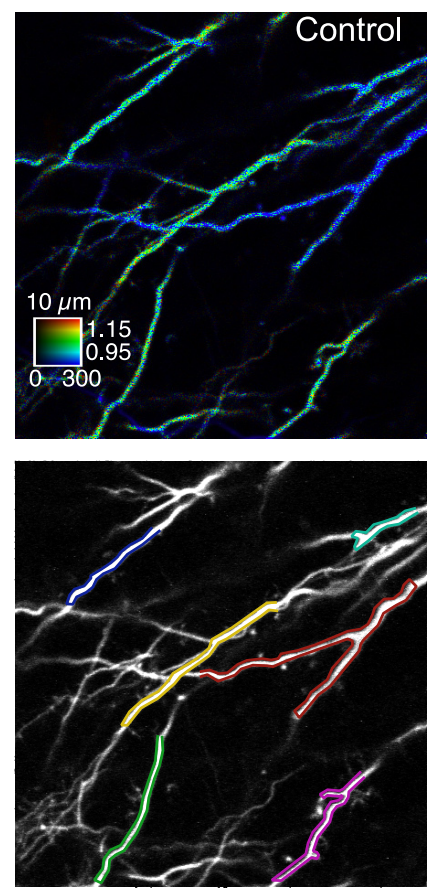

D
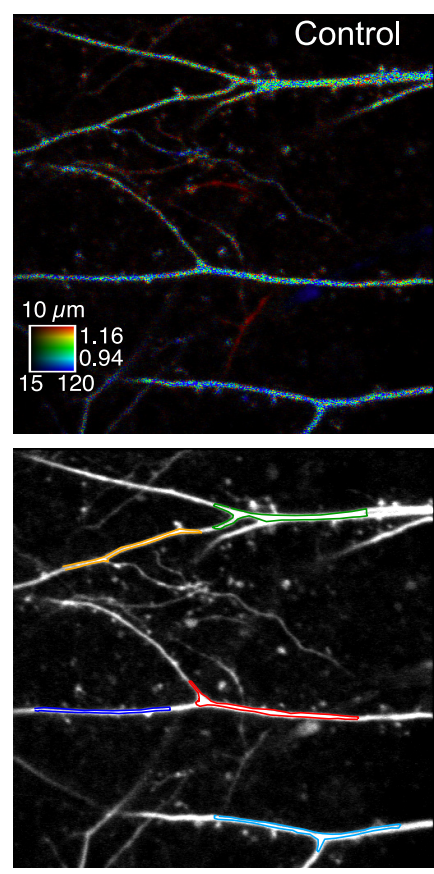

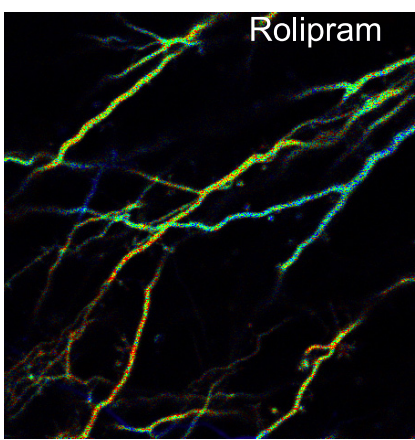

B
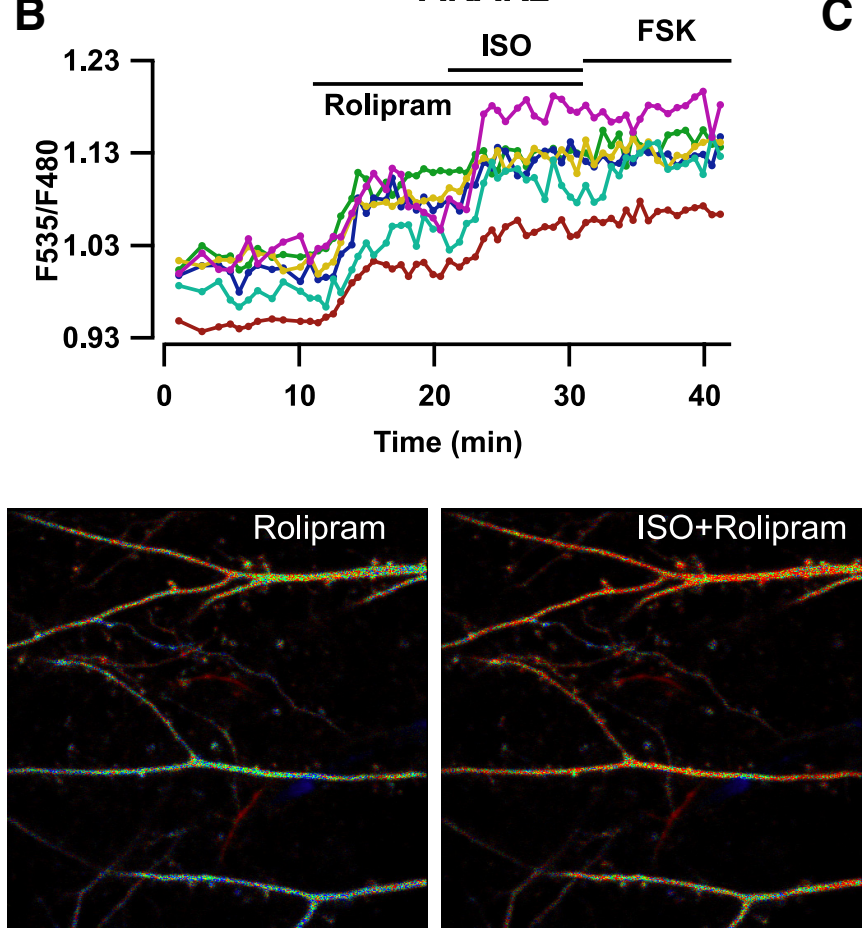

E

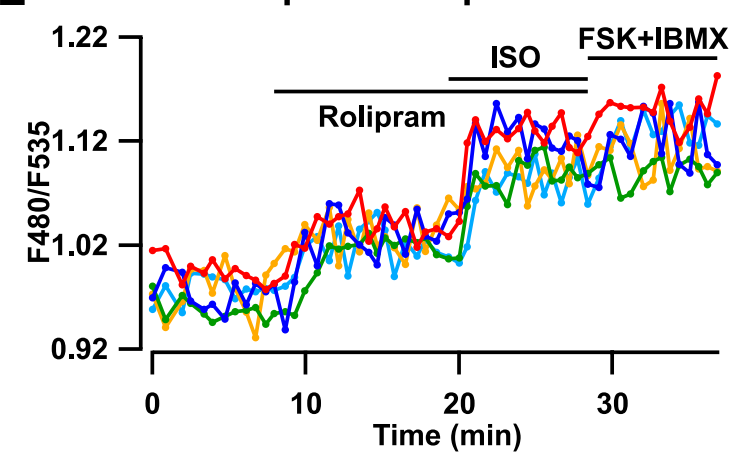

$\mathbf{F}$
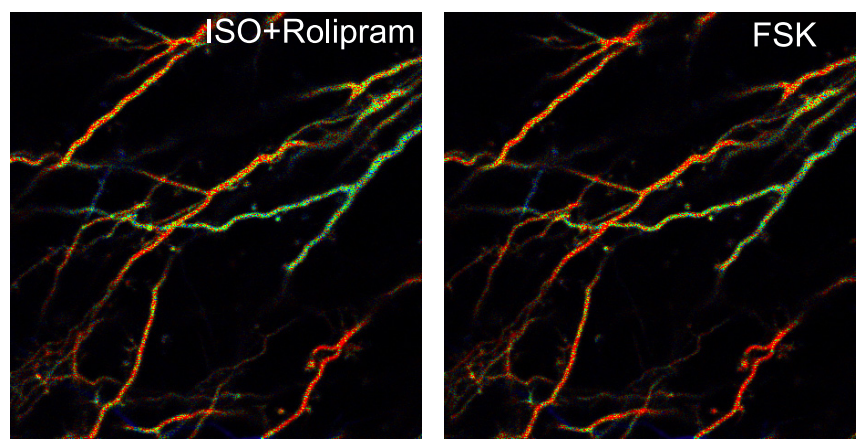

C

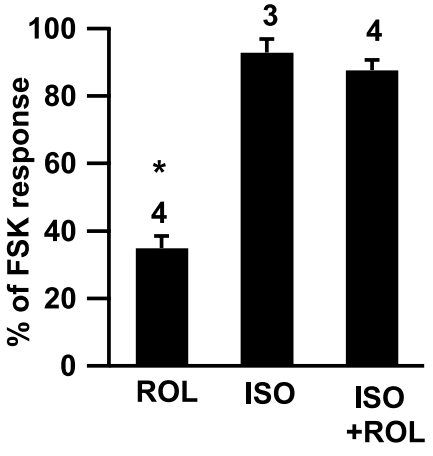

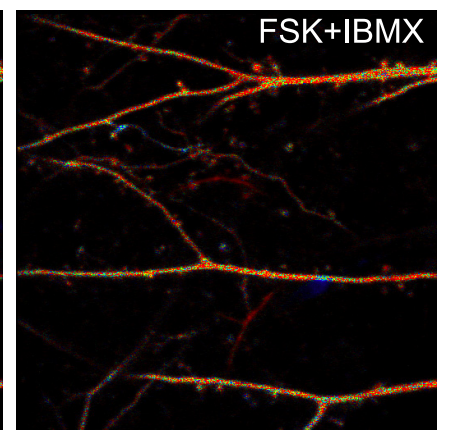

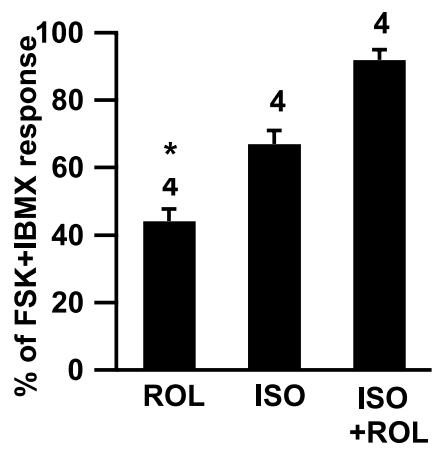

Figure 5. PDE4 inhibition with rolipram increases PKA activity and CAMP levels in thin apical dendrites in the parietal cortex. $\boldsymbol{A}-\boldsymbol{F}$, Pseudocolor images indicating AKAR2 (A) or Epac2-camps300-Cit (D) emission ratio in different experimental conditions. Each trace indicates the F535/F480 (B) or F480/F535 (E) emission ratio, measured on the regions indicated by the color contour on the grayscale image. Rolipram $(100 \mathrm{nM})$, ISO $(100 \mathrm{nM})$, FSK $(13 \mu \mathrm{M})$, and IBMX (200 $\mu \mathrm{M})$ were applied in the bath for the period of time indicated by the bars. The mean ratio change of AKAR2 $(\boldsymbol{C})$ and Epac2-camps300-Cit $(\boldsymbol{F})$ is expressed as a percentage of the maximal response obtained with FSK and FSK + IBMX, respectively. The asterisk indicates a statistically significant difference.

used as a reporter of PKA activation in this domain and PDE4 inhibition was shown to increase the PKA-dependent inhibition of tonic $I_{\mathrm{K}}$. In addition, the pharmacological inhibition of PKA doubled the tonic $I_{\mathrm{K}}$, showing that constitutive cAMP production is counterbalanced by PDE4 activity, resulting in intermediate levels of PKA activity. This steady-state level between tonic cAMP production and PDE4-mediated degradation was shown to critically affect neuronal excitability. These results were confirmed by visualizing the submembrane domain by two-photon imaging of biosensors in apical dendrites, a confined volume favoring 

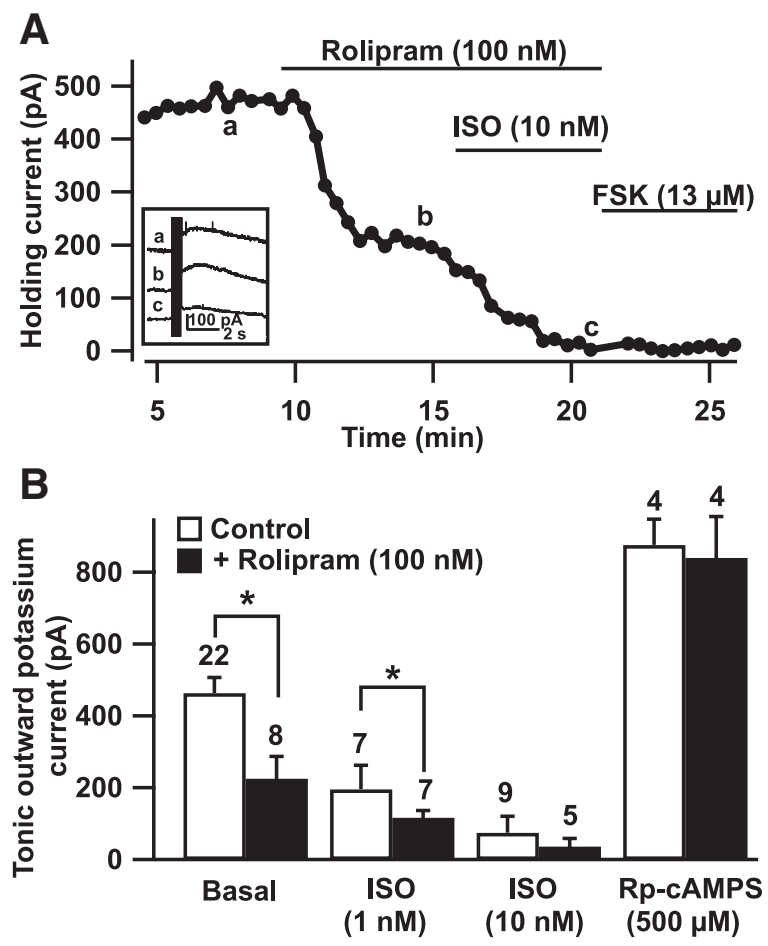

Figure 6. PDE4 inhibition with rolipram inhibits the tonic potassium current in a PKAdependent manner. $\boldsymbol{A}$, Suppression of tonic $I_{K}$ by rolipram, isoproterenol and FSK. The inset shows the individual SAHP traces obtained at the time points indicated by the corresponding letters on the main graph. $\boldsymbol{B}$, Effects of rolipram, ISO, and $\mathrm{R}_{\mathrm{p}}$-cAMPS (alone or combined) on tonic $I_{\mathrm{K}}$. The numbers of cells are indicated for each bar. Recordings were performed with $1 \mathrm{~mm}$ 5, 5' -dibromo BAPTA in the pipette solution. The asterisk indicates a statistically significant difference.

cAMP accumulation (see below). The results clearly showed that PDE4 inhibition alone increased tonic cAMP levels and PKA activity in this submembrane domain.

Functional differences in PKA signaling can result from the spatial organization of neurons, as was clearly reported in invertebrate neurons (Bacskai et al., 1993; Hempel et al., 1996). Our earlier work also showed that activation of the $5-\mathrm{HT}_{7}$ receptor in intralaminar thalamic neurons led to PKA responses that were both stronger and faster in the submembrane compartment (Gervasi et al., 2007). Differences between a submembrane domain and the bulk cytosol have also been reported in other cell types, including HEK293 cells (Rich et al., 2000, 2001) and cardiomyocytes (Saucerman et al., 2006). An important factor indeed is the diameter of the compartment: with cAMP production occurring at the membrane, a high surface area to volume ratio in small-diameter compartments favors the accumulation of cAMP, whereas the filling of a large cytosolic volume with cAMP, if ever possible, would take longer and be less efficient. Modeling approaches have suggested a threshold dendrite diameter of 1-3 $\mu \mathrm{m}$ to allow for cAMP accumulation (Neves et al., 2008). Our data obtained from morphologically intact neurons clearly fit to this model: cAMP accumulated in small dendrites, in which it was detected with Epac2-camps300-Cit and AKAR2. In larger domains such as the cell body, cAMP may still be produced at the same rate as at the membrane, but it rapidly diffuses throughout the large volume of the cell body, decreasing its concentration below the activation threshold for biosensors.

In addition to the geometric effects of surface area to volume ratio, the somatic and dendritic membranes of the neuron may
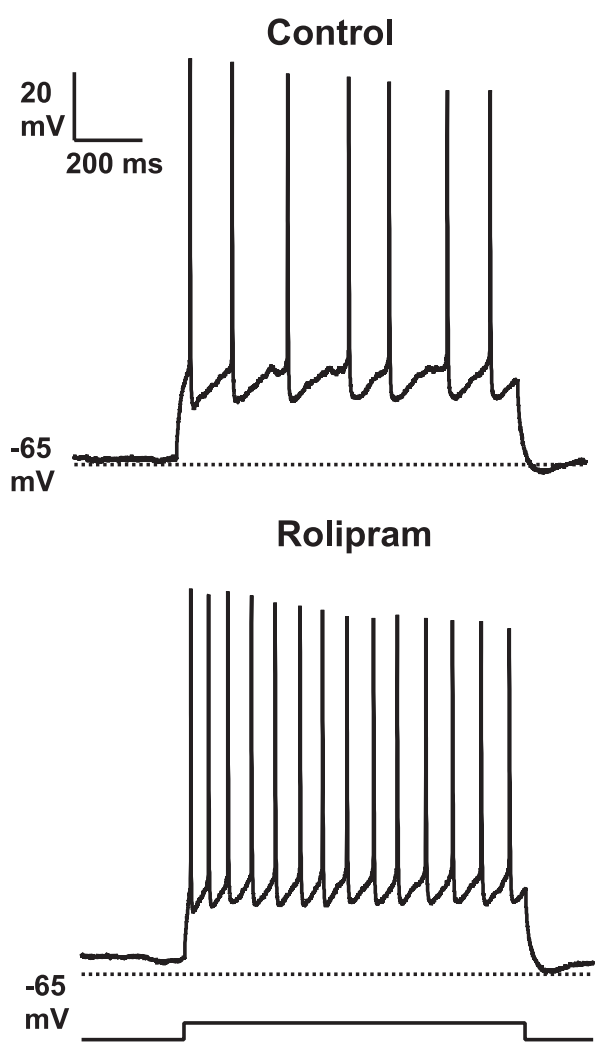

Figure 7. PDE4 inhibition increases spike frequency in pyramidal cortical neurons. Rolipram application doubled the spike frequency in response to 300 pA, 1 s current pulses. Recordings were performed with $1 \mathrm{~mm}$ EGTA in the pipette solution.

bear different types and/or concentrations of the proteins involved in the cAMP/PKA cascade. It remains unclear whether the compartment responsible for the signal detected by two-photon imaging and electrophysiology corresponds to the whole neuronal membrane, including the soma, or whether it is limited to the dendritic tree. Signaling microdomains formed by highly organized multiprotein complexes have also been shown to play an important role in this signaling (Tasken and Aandahl, 2004; Willoughby and Cooper, 2007). For example, PDE4 has been reported to interact with $\beta_{1}$-AR or to form a macromolecular complex with $\beta_{2}$-AR and $\beta$-arrestins in HEK293 cells and cardiomyocytes (Perry et al., 2002; Houslay and Baillie, 2005; Richter et al., 2008). Moreover, PDE4 is also known to interact with scaffolding proteins, such as A-Kinase Anchoring Proteins (AKAP), which have been shown to play a functional role in various cell types, including neurons (Rosenmund et al., 1994; Fraser and Scott, 1999; Tasken and Aandahl, 2004). However, intracellular perfusion with the AKAP decoy peptide Ht31 has no effect on the PKA-dependent modulation of sAHP current (Lancaster et al., 2006), indicating that AKAPs may not be involved in the regulation studied here. The properties of signaling microdomains can even be remodeled dynamically by the rapid translocation of key proteins involved in the signaling cascade. For example, type II regulatory subunits of PKA have been shown to be colocalized with MAP2 in the dendrites, thus maintaining a large pool of catalytic subunits for rapid release in response to noradrenergic stimulation, allowing the rapid diffusion of the free catalytic subunit into dendritic spines (Zhong et al., 2009). 


\section{Steady-state level of second messenger signals}

The results presented here reveal a peculiarity of these neuronal cells in the way they handle their intracellular cAMP concentration. We observed in the bulk cytosol that saturating doses of ISO failed to activate Epac1-camps, a probe with an $\mathrm{EC}_{50}$ for cAMP of $2.4 \mu \mathrm{M}$ (Nikolaev et al., 2004), while the same stimulus activated Epac2-camps300, a probe with an eightfold lower $\mathrm{EC}_{50}$ (Norris et al., 2009). This shows that free cAMP concentration clearly increases, but remains in the submicromolar range in the bulk cytosol during the $\beta_{1}$-adrenergic response. Modeling approaches have suggested that free cAMP concentration is $\sim 250 \mathrm{nM}$ in the soma during ISO stimulation, consistent with our observations (Neves et al., 2008). These values contrast findings for various other cell types, in which cAMP signals rise well above micromolar concentrations in response to various receptor agonists. For example, strong responses to $\beta$-adrenergic stimulation have been recorded with Epacl-camps in primary cultures of cardiomyocytes, fibroblasts, macrophages and thyroid cells (Leroy et al., 2008; Calebiro et al., 2009). Cell lines have also been reported to display strong responses, for example in the MIN6 pancreatic cell line after stimulation of the glucagon receptor (Landa et al., 2005 ), or in HEK-293 cells after stimulation of $\beta 2$-adrenergic receptors, where cAMP increases up to $10 \mu \mathrm{M}$ (Violin et al., 2008). cAMP degradation must therefore be particularly efficient in neurons, and we have shown that PDE4 is a key actor in this process. However, other mechanisms must also be at work, because, in the presence of rolipram, cAMP responses were far from saturating Epac2-camps300. These alternative cAMP degradation pathways may involve other PDEs, together with cAMP extrusion via multidrug resistance proteins (Wielinga et al., 2003).

Our results also demonstrate the occurrence of tonic cAMP production in the submembrane domain at a sufficiently high level for the tonic activation of PKA. This situation is reminiscent of the maintenance of steady-state cGMP levels in the submicromolar range in thalamic neurons by concomitant guanylyl cyclase and PDE2 activities (Hepp et al., 2007). Why would a cell waste ATP in such a 'futile cycle'? It is possible that tonic cAMP production and PKA activity provide more possibilities for signal integration and crosstalk with other cascades (Houslay and Milligan, 1997). Unlike an on/off system gated exclusively by Gs proteins, tonic activity allows both up- and downregulation, with $\mathrm{G}_{\mathrm{i}}$ proteins, phosphodiesterases and phosphatases all able to play a role. This situation is exemplified here for PDE4. The maintenance of this constitutive cAMP level throughout the bulk cytosol may be too costly for the neuron, and such tonic activity is therefore restricted to small dendrites, in which maximal flexibility is required to control neuronal excitability and the response to neurotransmitters.

\section{Toward a cellular model of the therapeutic efficacy of rolipram}

The cAMP/PKA signaling cascade is altered in several neuropsychiatric diseases (Wachtel, 1990; Duman et al., 1997; Perez et al., 2000). PKA has been evaluated as a target for the treatment of memory deficits, but the indiscriminate potentiation of PKA has been shown to have complex, and sometimes opposite, effects (Ramos et al., 2003; Arnsten et al., 2005). Previous studies have shown that PDE4 inhibition potentiates the $\beta_{1}$-AR response (Yamashita et al., 1997; H. T. Zhang et al., 2005): this effect possibly relates to the procognitive and antidepressant properties of the PDE4 inhibitor rolipram. Our experiments provide a mechanistic explanation, at the cellular level, for the effects of rolipram. In the bulk cytosol, the potentiating effect of rolipram on the
$\beta_{1}$-AR response was observed at low doses of agonist. Rolipram thus renders the neurons responsive to low agonist levels that would otherwise have little or no effect. These findings suggest that rolipram restores the postsynaptic response when the amount of neuromodulator released falls below the physiological detection threshold, thereby restoring normal function. In addition to its effect on the cytosolic integration of neuromodulatory responses, rolipram, when used alone, increases cAMP concentration and PKA activity at the membrane, thereby having a strong effect on the potassium current underlying the slow AHP. This results in global depolarization and an increase in the excitability of pyramidal cortical neurons. These two effects may account for the overall beneficial effect of rolipram, which compensates for pathologically low levels of neuromodulation and deficits in neuronal excitability (O'Donnell and Zhang, 2004).

\section{References}

Arnsten AF, Ramos BP, Birnbaum SG, Taylor JR (2005) Protein kinase A as a therapeutic target for memory disorders: rationale and challenges. Trends Mol Med 11:121-128.

Bacskai BJ, Hochner B, Mahaut-Smith M, Adams SR, Kaang BK, Kandel ER, Tsien RY (1993) Spatially resolved dynamics of cAMP and protein kinase A subunits in Aplysia sensory neurons. Science 260:222-226.

Calebiro D, Nikolaev VO, Gagliani MC, de Filippis T, Dees C, Tacchetti C, Persani L, Lohse MJ (2009) Persistent cAMP-signals triggered by internalized G-protein-coupled receptors. PLoS Biol 7:e1000172.

Duman RS, Heninger GR, Nestler EJ (1997) A molecular and cellular theory of depression. Arch Gen Psychiatry 54:597-606.

Ehrengruber MU, Lundstrom K, Schweitzer C, Heuss C, Schlesinger S, Gähwiler BH (1999) Recombinant Semliki Forest virus and Sindbis virus efficiently infect neurons in hippocampal slice cultures. Proc Natl Acad Sci U S A 96:7041-7046.

Fraser ID, Scott JD (1999) Modulation of ion channels: a "current" view of AKAPs. Neuron 23:423-426.

Gervasi N, Hepp R, Tricoire L, Zhang J, Lambolez B, Paupardin-Tritsch D, Vincent P (2007) Dynamics of PKA signaling at the membrane, in the cytosol, and in the nucleus of neurons in mouse brain slices. J Neurosci 27:2744-2750.

Goaillard, JM, Vincent P (2002) Serotonin suppresses the slow afterhyperpolarization in rat intralaminar and midline thalamic neurones by activating 5-HT7 receptors. J Physiol 541:453-465.

Haas HL, Konnerth A (1983) Histamine and noradrenaline decrease calcium-activated potassium conductance in hippocampal pyramidal cells. Nature 302:432-434.

Hempel CM, Vincent P, Adams SR, Tsien RY, Selverston AI (1996) Spatiotemporal dynamics of cAMP signals in an intact neural circuit. Nature 384:166-169.

Hepp R, Tricoire L, Hu E, Gervasi N, Paupardin-Tritsch D, Lambolez B, Vincent P (2007) Phosphodiesterase type 2 and the homeostasis of cyclic GMP in living thalamic neurons. J Neurochem 102:1875-1886.

Houslay MD, Baillie GS (2005) Beta-arrestin-recruited phosphodiesterase- 4 desensitizes the AKAP79/PKA-mediated switching of beta2adrenoceptor signalling to activation of ERK. Biochem Soc Trans 33:1333-1336.

Houslay MD, Milligan G (1997) Tailoring cAMP-signalling responses through isoform multiplicity. Trends Biochem Sci 22:217-224.

Lancaster B, Adams PR (1986) Calcium-dependent current generating the afterhyperpolarization of hippocampal neurons. J Neurophysiol 55:12681282.

Lancaster B, Batchelor AM (2000) Novel action of BAPTA series chelators on intrinsic $\mathrm{K}+$ currents in rat hippocampal neurones. J Physiol 522:231-246.

Lancaster B, Hu H, Gibb B, Storm JF (2006) Kinetics of ion channel modulation by cAMP in rat hippocampal neurones. J Physiol 576:403-417.

Landa LR Jr, Harbeck M, Kaihara K, Chepurny O, Kitiphongspattana K, Graf O, Nikolaev VO, Lohse MJ, Holz GG, Roe MW (2005) Interplay of $\mathrm{Ca}^{2+}$ and cAMP signaling in the insulin-secreting MIN6 beta-cell line. J Biol Chem 280:31294-31302.

Leroy J, Abi-Gerges A, Nikolaev VO, Richter W, Lechene P, Mazet JL, Conti 
M, Fischmeister R, Vandecasteele G (2008) Spatiotemporal dynamics of beta-adrenergic cAMP signals and L-type $\mathrm{Ca}^{2+}$ channel regulation in adult rat ventricular myocytes: role of phosphodiesterases. Circ Res 102:1091-1100.

McCormick DA, Prince DA (1986) Mechanisms of action of acetylcholine in the guinea-pig cerebral cortex in vitro. J Physiol 375:169-194.

McCormick DA, Williamson A (1989) Convergence and divergence of neurotransmitter action in human cerebral cortex. Proc Natl Acad Sci U S A 86:8098-8102.

Millar JK, Wilson-Annan JC, Anderson S, Christie S, Taylor MS, Semple CA, Devon RS, St Clair DM, Muir WJ, Blackwood DH, Porteous DJ (2000) Disruption of two novel genes by a translocation co-segregating with schizophrenia. Hum Mol Genet 9:1415-1423.

Millar JK, Pickard BS, Mackie S, James R, Christie S, Buchanan SR, Malloy MP, Chubb JE, Huston E, Baillie GS, Thomson PA, Hill EV, Brandon NJ, Rain JC, Camargo LM, Whiting PJ, Houslay MD, Blackwood DH, Muir WJ, Porteous DJ (2005) DISC1 and PDE4B are interacting genetic factors in schizophrenia that regulate cAMP signaling. Science 310: $1187-1191$

Millar JK, Mackie S, Clapcote SJ, Murdoch H, Pickard BS, Christie S, Muir WJ, Blackwood DH, Roder JC, Houslay MD, Porteous DJ (2007) Disrupted in schizophrenia 1 and phosphodiesterase 4B: towards an understanding of psychiatric illness. J Physiol 584:401-405.

Neves SR, Tsokas P, Sarkar A, Grace EA, Rangamani P, Taubenfeld SM, Alberini CM, Schaff JC, Blitzer RD, Moraru II, Iyengar R (2008) Cell shape and negative links in regulatory motifs together control spatial information flow in signaling networks. Cell 133:666-680.

Nguyen QT, Tsai PS, Kleinfeld D (2006) MPScope: a versatile software suite for multiphoton microscopy. J Neurosci Methods 156:351-359.

Nicoll RA (1988) The coupling of neurotransmitter receptors to ion channels in the brain. Science 241:545-551.

Nikolaev VO, Bünemann M, Hein L, Hannawacker A, Lohse MJ (2004) Novel single chain cAMP sensors for receptor-induced signal propagation. J Biol Chem 279:37215-37218.

Norris RP, Ratzan WJ, Freudzon M, Mehlmann LM, Krall J, Movsesian MA, Wang H, Ke H, Nikolaev VO, Jaffe LA (2009) Cyclic GMP from the surrounding somatic cells regulates cyclic AMP and meiosis in the mouse oocyte. Development 136:1869-1878.

O’Donnell JM, Zhang HT (2004) Antidepressant effects of inhibitors of cAMP phosphodiesterase (PDE4). Trends Pharmacol Sci 25:158-163.

Perez J, Tardito D, Mori S, Racagni G, Smeraldi E, Zanardi R (2000) Abnormalities of cAMP signaling in affective disorders: implication for pathophysiology and treatment. Bipolar Disord 2:27-36.

Perry SJ, Baillie GS, Kohout TA, McPhee I, Magiera MM, Ang KL, Miller WE, McLean AJ, Conti M, Houslay MD, Lefkowitz RJ (2002) Targeting of cyclic AMP degradation to beta 2-adrenergic receptors by beta-arrestins. Science 298:834-836.

Ramos BP, Birnbaum SG, Lindenmayer I, Newton SS, Duman RS, Arnsten AF (2003) Dysregulation of protein kinase a signaling in the aged prefrontal cortex: new strategy for treating age-related cognitive decline. Neuron 40:835-845.

Rich TC, Fagan KA, Nakata H, Schaack J, Cooper DM, Karpen JW (2000) Cyclic nucleotide-gated channels colocalize with adenylyl cyclase in regions of restricted cAMP diffusion. J Gen Physiol 116:147-161.

Rich TC, Fagan KA, Tse TE, Schaack J, Cooper DM, Karpen JW (2001) A uniform extracellular stimulus triggers distinct cAMP signals in different compartments of a simple cell. Proc Natl Acad Sci U S A 98:13049-13054.

Richter W, Day P, Agrawal R, Bruss MD, Granier S, Wang YL, Rasmussen SG Horner K, Wang P, Lei T, Patterson AJ, Kobilka B, Conti M (2008) Signaling from beta1- and beta2-adrenergic receptors is defined by differential interactions with PDE4. EMBO J 27:384-393.

Rosenmund C, Carr DW, Bergeson SE, Nilaver G, Scott JD, Westbrook GL (1994) Anchoring of protein kinase A is required for modulation of AMPA/kainate receptors on hippocampal neurons. Nature 368:853-856.

Sah P (1996) $\mathrm{Ca}(2+)$-activated $\mathrm{K}+$ currents in neurones: types, physiological roles and modulation. Trends Neurosci 19:150-154.

Sara SJ (2009) The locus coeruleus and noradrenergic modulation of cognition. Nat Rev Neurosci 10:211-223.

Saucerman JJ, Zhang J, Martin JC, Peng LX, Stenbit AE, Tsien RY, McCulloch AD (2006) Systems analysis of PKA-mediated phosphorylation gradients in live cardiac myocytes. Proc Natl Acad Sci U S A 19:2650-2658.

Tasken K, Aandahl EM (2004) Localized effects of cAMP mediated by distinct routes of protein kinase A Physiol Rev 84:137-167.

Vincent P, Gervasi N, Zhang J (2008) Real-time monitoring of cyclic nucleotide signaling in neurons using genetically encoded FRET probes. Brain Cell Biol 36:3-17.

Violin JD, DiPilato LM, Yildirim N, Elston TC, Zhang J, Lefkowitz RJ (2008) $\beta 2$-adrenergic receptor signaling and desensitization elucidated by quantitative modeling of real time cAMP dynamics. J Biol Chem 283: 2949-2961.

Wachtel H (1983) Potential antidepressant activity of rolipram and other selective cyclic adenosine $3^{\prime}, 5^{\prime}$-monophosphate phosphodiesterase inhibitors. Neuropharmacology 22:267-272.

Wachtel H (1990) The second-messenger dysbalance hypothesis of affective disorders. Pharmacopsychiatry 23:27-32.

Wielinga PR, van der Heijden I, Reid G, Beijnen JH, Wijnholds J, Borst P (2003) Characterization of the MRP4- and MRP5-mediated transport of cyclic nucleotides from intact cells. J Biol Chem 278:17664-17671.

Willoughby D, Cooper DM (2007) Organization and $\mathrm{Ca}^{2+}$ regulation of adenylyl cyclases in cAMP microdomains. Physiol Rev 87:965-1010.

Yamashita N, Yamauchi M, Baba J, Sawa A (1997) Phosphodiesterase type 4 that regulates cAMP level in cortical neurons shows high sensitivity to rolipram. Eur J Pharmacol 337:95-102.

Ye Y, O’Donnell JM (1996) Diminished noradrenergic stimulation reduces the activity of rolipram-sensitive, high-affinity cyclic AMP phosphodiesterase in rat cerebral cortex. J Neurochem 66:1894-1902.

Zhang HT, Huang Y, Jin SL, Frith SA, Suvarna N, Conti M, O'Donnell JM (2002) Antidepressant-like profile and reduced sensitivity to rolipram in mice deficient in the PDE4D phosphodiesterase enzyme. Neuropsychopharmacology 27:587-595.

Zhang HT, Huang Y, Mishler K, Roerig SC, O’Donnell JM (2005) Interaction between the antidepressant-like behavioral effects of beta adrenergic agonists and the cyclic AMP PDE inhibitor rolipram in rats. Psychopharmacology (Berl) 182:104-115.

Zhang J, Hupfeld CJ, Taylor SS, Olefsky JM, Tsien RY (2005) Insulin disrupts beta-adrenergic signalling to protein kinase A in adipocytes. Nature 437:569-573

Zhong H, Sia GM, Sato TR, Gray NW, Mao T, Khuchua Z, Huganir RL, Svoboda K (2009) Subcellular dynamics of type II PKA in neurons. Neuron 62:363-374. 